\title{
SCientific Oral Presentation AbStracts
}

Next, 55 teenage football players of the national U17 team were examined using a 1.5 Siemens Avanto Machine. The studies were interpreted by an MSK radiologist member of the AFC panel of radiologists who was aware of the FIFA scoring and grading system, as the ground truth. Thirty-six cases were used for training and 19 cases for testing of the CNN. To increase the number of training images, augmentation was performed by rotating and moving the original images. Therefore, a total number of 613 images were obtained for training and 267 images for testing. Results: Images introduced to the neural network resulted in sequential layers of meaningful output (Figure 2).

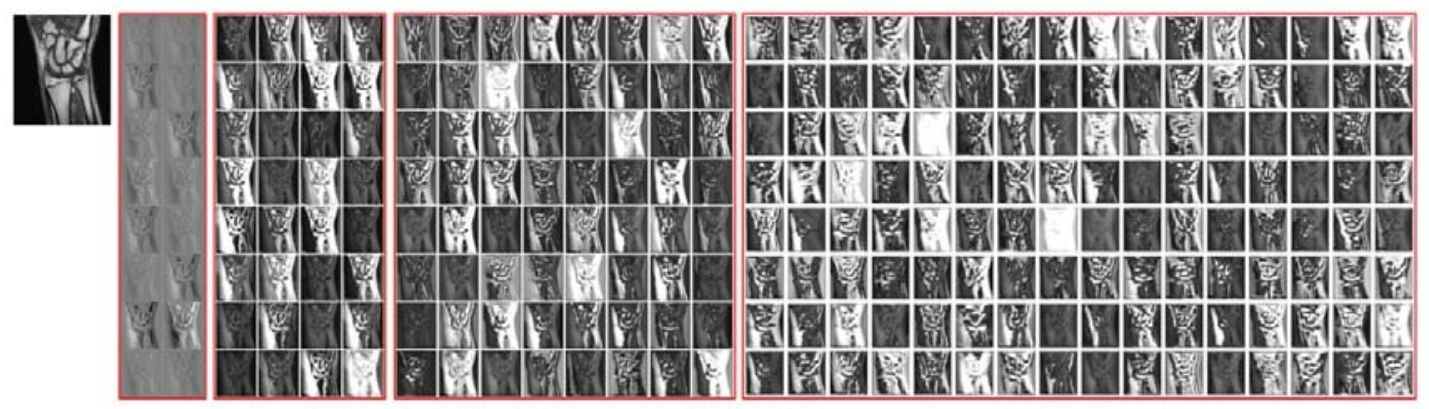

Figure 2. The resultant images in the CNN from the source image to the final FIFA grade

The final outcome of the network, as the FIFA grade of the case, was compared with the interpretation of the radiologist (Table 1). The findings indicated high accuracy of a single slice dataset while the accuracy approached $100 \%$ when the volumetric three slice sets were used.

\begin{tabular}{|c|c|c|}
\hline \multirow[t]{2}{*}{ Group } & \multicolumn{2}{|c|}{ Accuracy } \\
\hline & Single Middle Slice, $\%$ & Volumetric Three Middle Slices, \% \\
\hline Overall & 97.75 & 99.62 \\
\hline Grade II & 90 & 100 \\
\hline Grade III & 98.91 & 98.91 \\
\hline Grade IV & 97.91 & 100 \\
\hline Grade V & 97.43 & 100 \\
\hline Grade VI & 100 & 100 \\
\hline
\end{tabular}

Conclusion: The findings of this research indicated that CNN could be used for automatic bone age determination and FIFA grading of wrist MRI by reasonably high accuracy.

\section{References}

1. J.Dvorak, J.George, A.Junge, and J.Hodler “Age determination by magnetic resonance imaging of the wrist in adolescent male football players," BrJ Sports Med. 2007 Jan; 41(1): 45-52.

2. R.Malina, J.C.Eisenmann, S.P.Cumming. et al "Maturity-associated variation in the growth and functional capacities of youth football (soccer) players 13-15 years," Eur J Appl Physiol 2004.

3. H.Yousefi, M.Fatehi, M.Amian, R.A.Zoroofi “ A Fully Automated Segmentation of Radius Bone Based on Active Contour in Wrist MRI Data Set",20th Iranian Conference on Biomedical Engineering, Tehran, Iran, ICBME 2013.
4. H.Yousefi, M.Fatehi, M.Bahrami R.A.Zoroofi "3D Statistical Shape Models of Radius Bone for Segmentation in Multi Resolution MRI Data Sets", 21th Iranian Conference on Biomedical Engineering, Tehran, Iran, ICBME 2014

\section{-10.5812/IRANJRADIOL.99134}

\section{Epilepsy Presurgical Evaluation of Patients with Complex Source Localization by a Novel Component-Based EEG-fMRI Approach}

Elias Ebrahimzadeh" 2, 3, 4, *; Mohammad Shams ${ }^{5}$; Ali Rahimpour Jounghani ${ }^{6}$; Farahnaz Fayaz ${ }^{7}$; Mahya Mirbagheri'; Naser Hakimi $^{8,}{ }^{9}$; Seyed Sohrab Hashemi Fesharaki ${ }^{10}$; Hamid Soltanian-Zadeh ${ }^{1,11,12}$

${ }^{1}$ CIPCE, School of Electrical and Computer Engineering, College of Engineering, University of Tehran, Tehran, Iran

Artificial Intelligence in Medical Imaging. 2019 November 


\section{SCientific Oral Presentation Abstracts}

${ }^{2}$ Seaman Family MR Research Centre, University of Calgary, Calgary, Canada

${ }^{3}$ Department of Clinical Neurosciences, University of Calgary, Calgary, Canada

${ }^{4}$ Hotchkiss Brain Institute, Cumming School of Medicine, University of Calgary, Calgary, Canada

${ }^{5}$ Department of Biomedical Engineering, George Washington University, Washington D.C., United States

${ }^{6}$ Department of Psychological Sciences, University of California, Merced, United States

${ }^{7}$ Biomedical Engineering Department, School of Electrical Engineering, Payame Noor University of North Tehran, Tehran, Iran

${ }^{8}$ Division of Neonatology, Department of Pediatrics, UMCU-Wilhelmina Children's Hospital, Utrecht, Netherlands

${ }^{9}$ Artinis Medical Systems B.V., Elst, Netherlands

${ }^{10}$ Pars Advanced Medical Research Center, Pars Hospital, Tehran, Iran

${ }^{11}$ Image Analysis Laboratory, Department of Radiology, Henry Ford Hospital, Detroit, United States

${ }^{12}$ Department of Radiology, Wayne State University School of Medicine, Detroit, United States

${ }^{*}$ Corresponding author: CIPCE, School of Electrical and Computer Engineering, College of Engineering, University of Tehran, Tehran, Iran. Email:e_ebrahimzadeh@ut.ac.ir

\section{Abstract}

Background: The precise localization of epileptic foci is an unavoidable prerequisite for epilepsy surgery. Simultaneous EEG-fMRI recording has recently created new horizons to locate foci in patients with epilepsy and, in comparison with single-modality methods, has yielded promising results although it is still subject to a few limitations such as the lack of access to information between interictal events. This study assessed its potential added value in the presurgical evaluation of patients with complex source localization. Adult candidates considered ineligible for surgery on account of an unclear focus and/or presumed multifocality based on EEG underwent EEG-fMRI.

Objectives: Adopting a component-based approach, this study attempted to identify the neural behavior of the epileptic generators and detect the components of interest to be later used as inputs in the GLM model, substituting the classical linear regressor.

Methods: Nine IED sets from five patients were analyzed. These patients were rejected for surgery because of an unclear focus in two, presumed multifocality in one, and a combination of both in two of them.

Results: Component-based EEG-fMRI improved localization in three out of four patients with unclear foci. In patients with presumed multifocality, component-based EEG-fMRI advocated one of the foci in five patients and confirmed multifocality in one out of five patients. In two patients, component-based EEG-fMRI opened new prospects for surgery. In these complex cases, component-based EEG-fMRI either improved source localization or corroborated a negative decision regarding surgical candidacy.

Conclusion: As supported by the statistical findings, the developed EEG-fMRI method led to a more realistic estimation of localization than the conventional EEG-fMRI approach, making it a tool of high value in the presurgical evaluation of patients with refractory epilepsy.

\section{• 10.5812/IRANJRADIOL. 99135}

\section{Diagnostic Accuracy of Multi-Parametric Magnetic Resonance Imaging for Differentiation of Benign and Malignant Lesions of Prostate Using Radiomics Analysis}

SoheilaKoopaei ${ }^{1}$;AnahitaFathiKazerooni ${ }^{1}$;MahyarGhafoori ${ }^{2}$; Mohamadreza Alviri ${ }^{1}$; Kamal Hoseini ${ }^{1}$; Fakhereh Pashaei ${ }^{1}$; Hamidreza Saligheh $\operatorname{Rad}^{1, *}$

${ }^{1}$ Quantitative MR Imaging and Spectroscopy Group (QMISG), Research Center for Molecular and Cellular Imaging, Tehran University of Medical Sciences, Tehran, Iran

${ }^{2}$ Department of Radiology, Iran University of Medical Sciences, Tehran, Iran

*Corresponding author: Quantitative MR Imaging and Spectroscopy Group (QMISG), Research Center for Molecular and Cellular Imaging, Tehran University of Medical Sciences, Tehran, Iran. Email: hamid.saligheh@gmail.com

\section{Abstract}

Background: Prostate cancer is the second most common cancer-related cause of death in men. Accurate diagnosis of prostate cancer plays an important role in decreasing mortality rates. European Association of Urology (EAU) suggests multiparametric MRI (mp-MRI) of the prostate as a noninvasive method to evaluate prostate lesions. To leverage the interbreeder variability in the interpretation of mp-MRI, computer-aided diagnostic (CAD) systems can be used for automatic detection and characterization of prostate lesions.

Objectives: The goal of this article was to design a quantification method based on mp-MRI for the discrimination of benign and malignant prostatic lesions with MR imaging/transrectal ultrasonography fusion-guided biopsy as a reference for pathology validation.

Methods: Mp-MR images, including T1- and T2weighted, diffusion-weighted imaging (DWI), and dynamic contrast enhancement imaging (DCE) MRI were acquired at $1.5 \mathrm{~T}$ from 27 patients. Then, 106 ra- 